\title{
Multiscale Analysis of Delamination of Carbon Fiber- Epoxy Laminates with Carbon Nanotubes
}

\author{
JC Riddick ${ }^{*}$ \\ US Army Research Laboratory, Hampton VA 23681 \\ SJV Frankland ${ }^{\dagger}$ \\ National Institute of Aerospace, 100 Exploration Way, Hampton VA 23666 \\ TS Gates: \\ NASA Langley Research Center, Hampton, VA 23681
}

\begin{abstract}
A multi-scale analysis is presented to parametrically describe the Mode I delamination of a carbon fiber/epoxy laminate. In the midplane of the laminate, carbon nanotubes are included for the purposes of selectively enhancing the fracture toughness of the laminate. To analyze carbon fiber-epoxy-carbon nanotube laminate, the multi-scale methodology presented here links a series of parameterizations taken at various length scales ranging from the atomistic through the micromechanical to the structural level. At the atomistic scale molecular dynamics simulations are performed in conjunction with an equivalent continuum approach to develop constitutive properties for representative volume elements of the molecular structure of components of the laminate. The molecular-level constitutive results are then used in the Mori-Tanaka micromechanics to develop bulk properties for the epoxy-carbon nanotube matrix system. In order to demonstrate a possible application of this multi-scale methodology, a double cantilever beam specimen is modeled. An existing analysis is employed which uses discrete springs to model the fiber bridging affect during delamination propagation. In the absence of empirical data or a damage mechanics model describing the effect of CNTs on fracture toughness, several tractions laws are postulated, linking CNT volume fraction to fiber bridging in a DCB specimen. Results from this demonstration are presented in terms of DCB specimen load-displacement responses.
\end{abstract}

$\mathrm{W}$

ITH the explosive growth in the development of nanostructured materials, there has been established a need for multi-scale analysis methods that can be used to facilitate design and selection of materials for specific applications. For example, carbon nanotubes are known to possess the mechanical properties that, when used in conjunction with conventional structural materials such as carbon fiber composites, have the potential to enhance or contribute to increases in mechanical performance without the penalties of excessive weight gain. However, without advanced analysis methods, the multitude of synthesis and formulation options available to the material developer can lead to costly trial and error methods to determine the effectiveness of the nanomaterials to provide the required property enhancement. Therefore, to address the need for parametric tools capable of rapidly predicting bulk properties based on fundamental structural changes, Computational Materials or materials-by-design methods have been proposed. In particular, Gates, et al [1] have proposed a multi-scale analysis method that establishes connections between molecular, micro, and macro scales and relies on fundamental structure-property relationships. This approach provides a foundation for this paper.

\footnotetext{
* Aerospace Engineer, Vehicle Technology Directorate, AIAA Member.

${ }^{\dagger}$ Senior Research Scientist, AIAA Member.

${ }^{*}$ Head, Mechanics of Structures and Materials Branch, AIAA Associate Fellow.
} 
It is also recognized that near term applications of carbon nanotubes (CNT) in structural materials for aerospace vehicles are most likely to be realized through an approach that requires small quantities of nanotubes selectively used in conventional carbon fiber-polymeric composites. For example, carbon nanotubes added between the layers of a traditional carbon fiber-epoxy laminate may well improve the interface strength between carbon fiber and epoxy matrix, and thereby improve total fracture toughness of these laminates.

The effectiveness of this approach of selective enhancement can be analyzed with multi-scale models which are capable of incorporating molecular level details and then translating this information up the length scale to the bulk level where engineering properties are calculated. It is assumed that the primary focus of such analysis methods is to accurately describe behavior at the interfaces that occur between constituents at each scale level. At the atomisticlevel the assumptions of 'perfect' bonding between the various contributors to the laminate can be removed in detailed atomistic simulations. With properties at this level established, the micro-scale properties can be accessed with more standard micromechanics methods such as the Mori-Tanaka method or the rule-of-mixtures analysis [1,2] that assume perfectly bonded interfaces but allow for property gradients based on dispersion, orientation, or volume fraction of the major constituents.

Recent multi-scale models have applied atomistic simulation and micromechanics to the constitutive properties of various nanotube [3] and functionalized nanotube materials [4-6]. There are also some atomistic simulations of polymer-nanotube composites that address nanotube pull-out [7-8]. A more recent study on nanotubes chemically bonded into the epoxy matrix predicted a Young's modulus of up to $160 \mathrm{GPa}$ in the direction of the nanotube axis, and 4-8 GPa in the transverse direction at a nanotube volume fraction of $25 \%$ [9].

It is the objective of this paper to develop the interrelationships within a multi-scale analysis method that are capable of predicting the effect of single-walled CNT volume fraction on the elastic material parameters needed to calculate bulk fracture toughness of a carbon fiber-epoxy-CNT laminate. In lieu of experimentally-determined strength characteristics for carbon fiber-epoxy-CNT composites, critical damage parameters are postulated. For illustrative purposes, the standard double-cantilever beam (DCB), mode I fracture, type of test is considered with the laminate modified by selective application of CNT to the epoxy matrix in the mid-plane. Key structure-property relationships are established at each length scale and the relative influence of this intrinsic data is established at each scale. The methods are outlined and the utility of parametric studies are presented in the form of a bulk traction law and related load-displacements that would be typically measured during fracture testing. It is noted that due to the absence of available data on damage parameters at the laminate-level, no explicit conclusions can be drawn about the effect of CNT on the overall mode I laminate fracture toughness. The methodology developed here sets the stage for future high-fidelity analysis of selectively-nanostructured laminated composite materials to be performed based upon actual damage criteria arriving from experimental measurement.

\section{Material Description}

The material for the test case is a laminated composite composed of 24 layers (lamina) of unidirectional carbon fibers embedded in a graphite-epoxy matrix. The carbon fibers are 5 microns in radius and have a Young's modulus of $230 \mathrm{GPa}$ and a shear modulus of $17 \mathrm{GPa}$ [10]. At the mid-plane of the composite laminate, the matrix material includes $(10,10)$ single-walled carbon nanotubes of radius $0.678 \mathrm{~nm}$, such that the composite at the midplane may be characterized as a fiber-epoxy-CNT composite as shown in the exploded view in fig. 1. It is assumed that there is no chemical bonding between the 3 components: carbon fiber, carbon nanotubes, and the epoxy and therefore any changes in interface properties is due to non-bonded molecular interactions and macro-scale mechanical interlocking of the constituents.

\section{A. Test Case: Mode I Fracture}

The standard test method for establishing Mode I fracture toughness of polymer matrix composites (PMCs) is the DCB test [11]. Briefly, in the DCB test the laminate is loaded such that Mode I fracture occurs through delamination along the specimen midplane as shown in fig. 1. In this study, it is assumed that the matrix material in the midplane contains the nanostructured material applied directly to the PMC layers. Thus, in the case considered in this paper, the delamination in the laminate involves separation of layers along the midplane that also incorporate the epoxy-CNT layer. It should be noted that with regard to the Mode I fracture toughness of polymer matrix composites with carbon nanotubes, there is no available data for the critical values of Mode I fracture toughness. 


\section{Analysis Method}

The multi-scale analysis method used herein relies on key structure-property relationships at each scale level and establishing the interrelationships between these scale levels. The primary parameter or structural variable considered in this paper is the volume concentration of the CNT material. The approach is to vary the volume concentration over a reasonable range and predict material relationships that are required for engineering analysis of the DCB load-displacement response. The point of emphasis for the modeling at each scale level is the behavior at the respective material interface. The material is assumed to behave in a linear-elastic manner and elastic constants are calculated where required at each scale. Fracture is only considered at the macro-scale and hence CNT strength or bond-breaking at the molecular-scale are not considered in this study. At the ply-level interface fiber bridging is considered by calculating the strain energy release rate due to stresses with fiber pull-out.

\section{A. Constitutive Prpoerties}

The constitutive models require the definition of several representative volume elements (RVE) of the material. First, at the atomistic level, RVEs of the molecular structure of the epoxy, a nanotube in epoxy, and a nanotube in epoxy at the graphitic surface of the carbon fiber are generated with molecular dynamics (MD) simulations. This construct is termed the "effective fiber". The molecular RVEs are presented in fig 2. These MD simulations are in turn based on specific force-field parameterization relevant to the chemical structure of the components. The constitutive properties of the molecular RVEs are calculated using the equivalent continuum (EC) model [3] with the energies of deformation of the molecular structures determined from MD simulation [4].

The next level for which constitutive properties are required is the epoxy-nanotube composite in which carbon nanotubes are included in the matrix material of the midplane. The RVE at this scale has randomly dispersed high aspect ratio (1:1000) epoxy-nanotube effective fibers embedded in epoxy matrix. The constitutive properties are calculated as a function of nanotube volume with the Mori-Tanaka micromechanics method which is orientationally averaged [12-14]. Here the resulting constitutive properties are isotropic, and in this work, defined by a Young's and shear modulus.

At the lamina-level, constitutive properties are required for the fiber-epoxy laminae. The constitutive properties of the fiber-epoxy lamina are derived from the standard rule-of-mixtures assuming $60 \mathrm{vol} \%$ carbon fibers and epoxy properties from the MD/EC method using the RVE depicted in fig. 3(a). To facilitate the modeling of the Mode I delamination, the midplane will be modeled as a pair of neighboring lamina with CNT in the matrix material, constituting a carbon fiber-epoxy-CNT lamina. The constitutive properties for the fiber-epoxy-CNT lamina are calculated with the atomistically-informed rule-of-mixtures (AIRM) model. This model is a modification to the previously developed AIRM model given by [15],

$$
\begin{aligned}
& E_{L}=v_{f} E_{f}+\sum_{i}\left(v_{m} E_{m}\right)_{i}, \\
& \sum_{i}\left(v_{m} E_{m}\right)_{i}=v_{I} E_{I}+v_{I I} E_{I I} .
\end{aligned}
$$

Where, $v_{i}$ and $E_{i}$ are the volume fractions and Young's moduli, respectively. The subscript ' $L$ ' refers to the total longitudinal modulus corresponding to the 1-axis in fig. (1), the subscript ' $\mathrm{f}$ ' refers to the carbon fiber properties, ' $\mathrm{m}$ ' refers to the matrix properties. Note that the matrix properties are calculated by summing the properties of Regions I and II depicted in the lamina RVE in fig. (3). Region I, near the fiber surface, extends radially outward from the fiber for a fixed distance of 0.004 microns. The remaining volume of epoxy-nanotube material surrounding the carbon fiber constitutes Region II.

The fiber-epoxy-CNT laminate analyzed here is composed of 24 layers, where the matrix material of the innermost pair of lamina has a fixed volume fraction of carbon nanotubes. The laminate configuration constitutes a hybrid configuration where fiber-epoxy-CNT lamina properties are used for the pair of innermost layers and fiberepoxy lamina properties are used in the remaining lamina. The hybrid properties are calculated using classical lamination theory (CLT). The mechanical properties for the laminate may be estimated in terms of the constitutive properties of CLT as follows [16]:

$$
\begin{aligned}
& E_{11}=\frac{A_{11} A_{22}-A_{12}^{2}}{t A_{22}} \\
& E_{22}=\frac{A_{11} A_{22}-A_{12}^{2}}{t A_{11}}
\end{aligned}
$$




$$
v_{12}=\frac{A_{12}}{A_{22}}
$$

Here, $\mathrm{A}_{\mathrm{ij}}$ are the extensional stiffness of the laminate, $\mathrm{t}$ is the thickness, $\mathrm{E}_{11}$ and $\mathrm{E}_{22}$ are the elastic moduli of the laminate in the 1-2 plane shown in fig. (1), and $v_{12}$, the Poisson's ratio in the 1-2 plane.

\section{B. Load-Displacement Curves}

Having calculated the constitutive properties of the fiber-matrix system and its components, if a criterion for damage of fiber-epoxy-CNT laminates exists, the load-displacement curves during Mode I delamination response can be calculated using an analysis of a DCB specimen such as the one depicted in fig. (4a). By postulating a conditions for carbon fiber being pulled out of the epoxy-nanotube matrix based upon an elastic analysis, a traction law is postulated for the pull-out of the carbon fiber. The assumed traction law representing fiber pull-out is used in a load-displacement calculation for Mode I delamination [17] in which the bridging carbon fibers that bridge the crack are represented as discrete springs oriented normal to the crack plane and the overall geometry of the delamination specimen is included.

Here, the traction law is characterized by a load-displacement relationship which exhibits a monotonic increase in load up to a critical value at the initial displacement of the fiber across the delamination opening as shown in fig. (4a). The initial displacement $z_{0}$, which is half of the crack opening displacement, arises from the initial loading of the DCB specimen up to the initiation of the delamination. Upon initial loading, the fiber displaces monotonically until this critical displacement is reached at peak load, $P_{c}$, exhibiting a constant loading stiffness $k_{o}$. The peak load corresponds to the load at which the assumed pull-out condition is achieved for a single fiber.

As the fiber displaces beyond $z_{o}$, thus undergoing pull-out, the stiffness decreases linearly with displacement until complete pull-out is reached at $z_{f}$ and the fiber stiffness becomes zero. Using this postulated damage model, the stiffness of the bridging fiber $k_{i}$ is [17]

$$
k_{i}= \begin{cases}k_{o} & \Leftarrow z_{i} \leq z_{o} \\ (1-d) k_{o} & \Leftarrow z_{o} \leq z_{i} \leq z_{f} \\ 0 & \Leftarrow z_{i} \geq z_{f}\end{cases}
$$

where $z_{o}$ is the fiber displacement corresponding to the condition at which the critical debond length is reached, and $z_{\mathrm{f}}$ is the displacement at which the fiber stiffness becomes zero, corresponding to complete pull-out. The value $\mathrm{z}_{\mathrm{f}}$ is the total of the fiber displacement $\mathrm{u}$ and the critical debond length $\mathrm{L}_{\mathrm{d} 1}$. The damage parameter, $\mathrm{d}$, is given by [17]:

$$
d=\frac{z_{f}\left(z_{i}-z_{o}\right)}{z_{i}\left(z_{f}-z_{o}\right)}
$$

The traction laws are calculated for different volume fractions of carbon nanotubes in epoxy by postulating conditions for debonding and pull-out based upon an elastic analysis to be described later.

The load-displacement curve for Mode I delamination is then calculated using a special-purpose code [17] in which the carbon fibers are represented by discrete springs pulled out of the matrix. In the present analysis the properties for the epoxy-nanotube matrix system derived using the AIRM are incorporated into the traction law via an elastic analysis of strain energy release rate associated with fiber pull-out which will be detailed later. The discrete springs (or carbon fibers) are arranged in 42 rows each having 10 springs. The springs are attached to an idealized cantilever beam which has the constitutive properties of the top 12 layers of the laminate as deterrmined from the CLT analysis. The geometry of the model laminate specimen to be delaminated is equivalent to that of a double cantilever beam (DCB) specimen which is $120 \mathrm{~mm}$ in length and $20 \mathrm{~mm}$ in width as diagrammed in fig 5 . The specimen has an initial crack opening displacement of $20 \mathrm{~mm}$. The specimen laminate is loaded such that Mode I delamination occurs at the midplane as shown in fig. 1, i.e. the innermost lamina peel apart as the laminate is loaded. Thus, the delamination in the laminate involves separation of layers with CNT between them. The loaddisplacement response curves are parameterized by varying carbon nanotube concentrations in the midplane layer of up to $15 \mathrm{vol} \%$.

\section{Fiber Pull-out}

In the absence of available data for the critical strength values of fiber-epoxy-CNT material systems, the present analysis seeks to provide a potential methodology by which to quantify the effect of CNT on the Mode I delamination response of selectively-reinforced polymer composites. In lieu of measured values for damage criteria, 
an elastic analysis for fiber pull-out [18] is used to calculate the strain energy release rate associated with the critical debond length of a bridging carbon fiber.

The energy release rate $\mathrm{G}$ is calculated by solving stress equilibrium equations for a fiber that exhibits debonding when exposed to a pull-out stress, $\sigma_{b}$, with interfacial shear stress, $\tau_{s}$, defined at the fiber matrix interface as idealized in fig. 4. Using minimum potential energy principles, an expression for energy release rate, G, associated with the debonding of a fiber from the matrix has been derived such that [18]

$$
\begin{aligned}
G= & -\frac{1}{2 \pi r_{1}} \cdot \frac{\partial \Pi}{\partial L_{d}}=\lambda_{1} L_{d}^{2}+\lambda_{2} L_{d}+\lambda_{3}, \\
\lambda_{1}= & \frac{E_{c} \tau_{s}^{2}}{r_{1} E_{f} V_{m} E_{m}}, \\
\lambda_{2}= & -\frac{\rho \lambda^{2} r_{1} \beta\left(1+v_{m}\right) \tau_{s}^{2}}{4 E_{m}}+\frac{3 E_{c} \tau_{s}^{2}}{r_{1} \beta E_{f} V_{m} E_{m}}-\frac{\sigma_{b} \tau_{s}}{E_{f}}, \\
\lambda_{3}= & \frac{\rho r_{1}^{2} \lambda^{2} \beta V_{m}\left(1+v_{m}\right) \sigma_{b} \tau_{s}}{8 E_{c}}-\frac{3 \sigma_{b} \tau_{s}}{2 E_{f} \beta}+\frac{r_{1} E_{m} V_{m} \sigma_{b}^{2}}{4 E_{c} E_{f}} \\
& +\frac{r_{1} \phi \tau_{s}^{2}}{2 G_{m}}-\frac{\lambda^{2} \tau_{s}^{2}}{8 r_{1}^{3} G_{m}}\left[4 r_{2}^{4} \ln \left(r_{1} / r_{2}\right)+4 r_{1}^{2} r_{2}^{2}-r_{1}^{4}\right. \\
& \left.-3 r_{2}^{4}\right]-\frac{r_{1} \tau_{s}}{8 G_{f}} .
\end{aligned}
$$

where, $r_{1}$ is the fiber radius, $L_{d}$ is the debond length, $E_{i}$ refers to the Young's modulus, $G_{i}$ to the shear modulus, and $v_{i}$ to the Poisson's ratio, the subscipt ' $m$ ' refers to matrix properties, and the subscript ' $\mathrm{f}$ ' refers to fiber properties. Note that here $\mathrm{E}_{\mathrm{m}}$ is determined using the AIRM described above allows the effect of the properties at the molecular scale to be incorporated into the fiber bridging analysis. The parameters $\rho, \beta, \mathrm{E}_{\mathfrak{c}}$, and $\lambda$ are defined as follows in terms of the parameters included in the schematic of fig. 4 [18]:

$$
\begin{aligned}
& \rho=\frac{4}{V_{f}^{2}} \ln \left(r_{1} / r_{2}\right)-\frac{3}{V_{f}^{2}}+\frac{4}{V_{f}}-1, \\
& \beta^{2}=\frac{4 \alpha+\lambda}{\rho \lambda^{2} r_{1}^{2}\left(1+v_{m}\right)}, \\
& E_{c}=V_{f} E_{f}+V_{m} E_{m}, \\
& \lambda=\frac{V_{f}}{V_{m}} .
\end{aligned}
$$

where $\mathrm{V}_{\mathrm{i}}$ refers to volume fraction. For a known set of geometry and material parameters, the strain energy release rate $\mathrm{G}$ is a quadratic function of $\mathrm{L}_{\mathrm{d}}$.

To form the traction laws described above some critical values must be established here to correspond with the peak load $\mathrm{P}_{\mathrm{c}}$, initial fiber displacement $\mathrm{z}_{\mathrm{o}}$, and the final fiber pull-out displacement $\mathrm{z}_{\mathrm{f}}$. By assuming a constant value for critical debond length $\mathrm{L}_{\mathrm{d} 1}$, for all variations of CNT volume concentration, a parameterization of the Mode I fracture results may be performed. The critical debond length may be calculated using the following expression [18]:

$$
L_{d 1}=\frac{-\lambda \pm \sqrt{\lambda_{2}^{2}-4 \lambda_{1}\left(\lambda_{3}-\Gamma\right)}}{2 \lambda_{1}} .
$$

where $\Gamma$ is the critical strain energy release rate for a given for a given volume concentration.

Finally, the initial fiber displacement as depicted in fig. 4, represented by the variable $u$ is given in terms of critical debond length $\mathrm{L}_{\mathrm{d} 1}$ by the expression [18]

$$
u=\frac{E_{m} V_{m}}{E_{c}} u_{o},
$$




$$
u_{o}=-\frac{\tau_{s} E_{c}}{r_{1} E_{f} E_{m} V_{m}} L_{d 1}^{2}+\left(\frac{\sigma_{b}}{E_{f}}-\frac{2 \tau_{s} E_{c}}{r_{1} \beta E_{f} E_{m} V_{m}}\right) L_{d 1}+\frac{\sigma_{b}}{\beta E_{f}} .
$$

For the purposes of the present study, the traction law for a single bridging fiber may be assumed numerically. In this case, the initial fiber displacement $z_{0}$ corresponds to the fiber displacement $u$, the final fiber pull-out displacement $\mathrm{z}_{\mathrm{f}}$ is the sum of initial fiber displacement $\mathrm{u}$ and the critical debond length $\mathrm{L}_{\mathrm{d} 1}$ and the peak load can be determined using the strain energy parameter $\Gamma$.

\section{Results and Discussion}

Results for the elastic constants of the molecular RVEs considered are listed in Table 1. The data listed are the results of predicted by the MD simulations and EC model described earlier. The elastic constants listed for the epoxy-CNT RVE are used in Mori-Tanaka micromechanics to yield an effective Young's modulus of the epoxy matrix with randomized effective epoxy-CNT fibers. Constants for $0 \%, 1.5 \%, 5 \%$, and $15 \%$ CNT volume fraction are listed in Table 2. Here the CNT volume fractions refer to the volume percent of effective CNT fibers in the nanocomposite epoxy-nanotube system considered.

The AIRM model was used to calculate the effective modulus of the total volume of matrix. Two regions are defined within the matrix relative to the carbon fiber as shown in fig. 3. For Region I, near the carbon fiber surface, constitutive properties derived directly from the atomistic simulation. The carbon nanotube-epoxy-graphite elastic constants from Table 1 are listed. For the remaining volume of matrix, Region II, effective properties listed in Table 2 are used in the analysis. The results from the AIRM analysis for various CNT volume fractions are listed in Table 3.

Referring to fig. 6 , strain energy release rate $G$ versus normalized debond length $L_{d}$ results illustrate that the strain energy release rate is a strong function of the volume concentration of carbon nanotubes. In particular, for the range of carbon nanotube concentrations considered, the chosen values of stress, and for a constant debond length, it was found that as carbon nanotube volume fraction increases the strain energy release rate increases even for small $(1.5 \%)$ volume concentrations. For example, by designating the normalized debond length value of 20 , indicated by the vertical dotted line in the fig. 6 , it is predicted that the value of strain energy release rate, here defined as $\Gamma$, decreases as volume fraction increases. Additionally, for a given strain energy release rate value, as carbon nanotube concentration increases the normalized debond length also increases.

To demonstrate the utility of parametric studies, Mode I delamination parameters are calculated using the multiscale constituent values. Traction laws governing the load-displacement of fibers during pull-out are postulated by considering the debond length corresponding to the normalized debond length of 20 , as designated above. For the CNT volume fractions considered here, the corresponding strain energy release rate $\Gamma$ is used to calculate the peak load in the traction law, defined earlier. The following sections describe the Mode I delamination parametric study.

Figure 7 illustrates provides the calculated traction laws for a range of CNT volume concentrations. The results in fig. 7 indicate the relative effect of volume concentration on the traction law of a single fiber. As the carbon nanotube volume concentration increases, there is a corresponding increase in the initial fiber displacement and the critical load. While the debond length remains constant, the final pull-out displacement increases with the increase in CNT volume fraction corresponding to the increase in initial fiber displacement. Therefore, for the parameters selected, as CNT volume fraction increases the area under the traction law response curve increases.

To consider the effect on the response of a DCB specimen resulting from adding carbon nanotubes to the innermost layers of a laminated carbon fiber composite, the load-displacement responses of a DCB specimens 120 $\mathrm{mm}$ in length and $20 \mathrm{~mm}$ in width were calculated using the postulated traction laws. Here the Mode I delamination analysis was carried out on a DCB specimen where 42 rows of bridging fibers, each row having 10 fibers, are idealized as discrete springs as shown in fig. 5(a). The load-displacement response curves shown in fig. 8 for a range of carbon nanotube concentrations are characterized by an initial loading phase, followed by a small, but noticeable, developing phase, and finally by a steady-state phase of stable crack growth with fiber bridging. The results predict that as carbon nanotube concentration increases, the load-displacement response will reflect an increase in load for a given displacement. The separation in the responses develops during the initiation phase of the loading and propagates as the displacement grows.

\section{Summary and Conclusions}

A multi-scale analysis has been conducted to analyze aspects of the Mode I delamination of unidirectional laminated carbon fiber-epoxy laminates with carbon nanotubes in the matrix material of the midplane layers. In particular, the constitutive behavior reflects an altering of the matrix properties by varying the CNT volume 
concentration. The resultant behavior on single fiber pull-out is calculated using an assumed interfacial shear stress and pull-out stress. To demonstrate the structure-property relationship at each scale level, an example problem is provided that calculates the macro-scale laminate fracture toughness in terms changes in intrinsic molecular properties. Mode I fracture or delamination is studied by predicting the effects of a series of parameterizations taken at various length scales ranging from the atomistic through the micromechanical to the structural scale.

In terms of scales addressed in this paper, the atomistic level results of molecular dynamics simulations are used within an equivalent-continuum method to develop elastic properties and are subsequently used in a micromechanics analysis method. The micromechanical analysis yields a set of effective elastic properties for the matrix with carbon nanotubes. The effective properties for the matrix near the carbon fiber, within 0.004 microns, are taken directly from the $\mathrm{MD} / \mathrm{EC}$ model. Elastic properties for a single ply or lamina with carbon nanotubes in the matrix are then developed using an atomistically-informed rule-of-mixtures (AIRM) that allows the matrix proeprites near a carbon fiber to vary according to assumed changes in the concentration of carbon nanotubes as you proceed outward from the fiber surface. Laminate level properties are generated using standard classical lamination theory.

Is it expected that fracture toughness of the laminate may well be enhanced by fiber bridging due to fiber pullout. To assess the influence of adding carbon nanotubes on the fiber bridging effects, a fiber pull-out model was employed which relies upon an equilibrium-based solution and an assumed interfacial shear stress and a pull-out stress. The results show that for interfacial shear stress and pull out stress equal to $10 \mathrm{MPa}$ and $1 \mathrm{GPa}$ respectively, and a constant normalized debond length of 20 , the strain energy release rate increases as the CNT volume concentration increases. The implication here is that as the matrix is made stiffer by the addition of carbon nanotubes the strain energy required to initiate fiber pull out increases.

To demonstrate the effect of the presence of carbon nanotubes near the midplane on the overall laminate fracture toughness, a discrete spring model for predicting delamination growth was used to perform a DCB specimen analysis. The DCB analysis incorporated the properties derived from the multi-scale analysis. Thus, the results of the discrete spring model are influenced by the molecular-level properties of the epoxy-carbon nanotube material. In order to perform the DCB analysis traction laws were postulated for bridging fibers based upon the stresses, debond length and strain energy release rate. The traction law results presented herein reflect the fact that for a constant debond length, as carbon nanotube concentration is increased, there is a corresponding increase in the initial fiber displacement, the final pull-out displacement, and the critical load. Here, as carbon nanotube volume fraction increases the area under the traction law response curve increases implying the localized effect of the elastic constitutive properties of the carbon nanotubes in the matrix may well influence bulk laminate fracture toughness in the presence of fiber bridging.

The calculated load-displacement response curves indicate that as carbon nanotube concentration is increased, the bulk load-displacement relationship varies. The initiation development phases of the loaddisplacement curves coincide over a range of carbon nanotube concentrations. However, as the phase of steady-state crack growth develops, there is an increase in the load for a given value of displacement, as carbon nanotube volume fraction in the innermost lamina increases from $1.5 \%$ to $15 \%$ volume fraction. The implication here is that for a given crack opening displacement more load is required to achieve the opening as the carbon nanotube concentration increases. This result is directly attributable to the difference in the initial fiber displacement which is dependent on the matrix properties. The multi-scale analysis presented indicates a need for the experimental measurement of strength data for fiber-epoxy-CNT composites to validate the model and to provide the structureproperty relationships between localized application of carbon nanotubes amd laminate behavior during fracture.

\section{References}

[1] Gates, TS; GM Odegard; SJV Frankland; and TC Clancy; Composite Science and Technology, 65, 2416 (2005).

[2] Frankland, SJV; VM Harik; GM Odegard; DW Brenner; TS Gates; Composite Science and Technology, 63, 1655 (2003).

[3] Odegard, GM; TS Gates; RE Wise; C Park; EJ Siochi; Composite Science and Technology, 63, 1671 (2003).

[4] Frankland, SJV; GM Odegard; and TS Gates; AIAA Journal, 43, 1828, (2005).

[5] Odegard, GM; SJV Frankland; MN Herzog; TS Gates; and CC Fay; "Constitutive Modeling of Cross-Linked Nanotube Materials", AIAA-2004-1606, 45 ${ }^{\text {th }}$ AIAA/ASME/ASCE/AHS/ASC Structures, Structural Dynamics, and Materials Conference, Palm Springs, CA, (2004).

[6] Gates, TS; GM Odegard; MP Nemeth; and SJV Frankland, "Predicting the Influence of Nano-scale Material Strucutre in the In-plane Buckling of Orthotropic Plates", AIAA-2004-1607, 45 ${ }^{\text {th }}$ AIAA/ASME/ASCE/AHS/ASC Structures, Structural Dynamics, and Materials Conference, Palm Springs, CA, (2004). 
[7] Gou, J; Z Liang; C Zhang; and B Wang; Composites B, 36, 524 (2005).

[8] Frankland, SJV and VM Harik; "Analysis of Carbon Nanotube Pull-out from a Matrix", NASA/CR-2002211743, NASA-Langley Research Center, Hampton, VA (2002).

[9] Clancy, TC and TS Gates; Mechanical Properties of Nanostructured Materials Determined Through Molecular Modeling Techniques", $46^{\text {th }}$ AIAA/ASME/ASCE/AHS/ASC Structures, Structural Dynamics, and Materials Conference, Austin, TX, (2005).

[10] Chung, DDL; Carbon Fiber Composites, Butterworth-Neinemann, Boston, MA (1994).

[11] ASTM Test Method D 5528-01, "Standard Test Method for Interlaminar Fracture Toughness of Unidirectional Fiber-Reinforced Polymer Matrix Composites", Annual Book of ASTM Standards, vol. 15.03, American Society of Testing and Materials, West Conshohocken, PA (2001).

[12] Mori,T and K Tanaka; Acta Metallurgica, 21, 571 (1973).

[13] Benveniste, Y; Mechanic of Materials, 6, 147 (1987).

[14] Berryman, JG; Journal of the Acoustical Society of America, 68, 1820 (1980).

[15] S. J. V. Frankland, J. C. Riddick and T. S. Gates, "Multi-scale Rule-of-Mixtures Model of Carbon Nanotube/Carbon Fiber/Epoxy Lamina", in Degradation Processes in Nanostructured Materials, ed., M. Chipara, O. Puglisi, R. Skomski, F. Jones, B.S. Hsiao, Material Research Society Symposium Proceedings, Volume 887, Q1.2 (2005).

[16] Jones, RM, Mechanics of Composite Materials, Taylor and Francis, New York, NewYork 1998.

[17] Ratcliffe, JG and TK O'Brien; "Discete Spring Model for Prediciting Delamination Growth in Z-Fiber Reinforced DCB Specimens", NASA/TM-2004-213019 (ARL-TR-3190), NASA-Langley Research Center, Hampton, VA (2004).

[18] Liu, P; W Tao; Y Guo; Journal of Zhiejang University SCIENCE, 6A (Suppl. I), 8, (2005).

\section{Tables and Figures}

Table 1- The Elastic Constants of the Molecular RVEs.

\begin{tabular}{|c|c|c|}
\hline Elastic Constants & $\begin{array}{c}\text { Epoxy/Nanotube } \\
(\mathbf{G P a})\end{array}$ & $\begin{array}{c}\text { Epoxy/Nanotube/Graphite } \\
(\mathbf{G P a})\end{array}$ \\
\hline $\mathrm{C}_{11}$ & 99.0 & 267 \\
\hline $\mathrm{C}_{22}$ & 9.43 & 182 \\
\hline $\mathrm{C}_{33}$ & 6.22 & 9.94 \\
\hline $\mathrm{K}_{12}$ & 31.9 & 138 \\
\hline $\mathrm{K}_{13}$ & 32.4 & 72.2 \\
\hline $\mathrm{K}_{23}$ & 12.1 & 49.5 \\
\hline $\mathrm{G}_{12}$ & 0.33 & \\
\hline $\mathrm{G}_{13}$ & 0.32 & \\
\hline $\mathrm{G}_{23}$ & 0.20 & \\
\hline
\end{tabular}


Table 2 - The Effective Elastic Properties of the Matrix with Carbon Nanotubes.

\begin{tabular}{|c|c|c|}
\hline $\begin{array}{c}\text { Carbon Nanotube } \\
\text { Volume Fraction } \\
(\%)\end{array}$ & $\begin{array}{c}\text { Matrix Effective } \\
\text { Young's } \\
\text { modulus } \\
\text { (GPa) }\end{array}$ & $\begin{array}{c}\text { Matrix Effective } \\
\text { Shear modulus } \\
(\mathbf{G P a})\end{array}$ \\
\hline 0 & 1.44 & 0.51 \\
\hline 1.5 & 2.69 & 0.96 \\
\hline 5 & 5.70 & 2.08 \\
\hline 15 & 16.92 & 6.31 \\
\hline
\end{tabular}

Table 3 - The Effective Elastic Properties of the Matrix in Fber-Epoxy-CNT Lamina.

\begin{tabular}{|c|c|c|c|}
\hline $\begin{array}{c}\text { Carbon Nanotube } \\
\text { Volume Fraction } \\
\mathbf{( \% )}\end{array}$ & $\begin{array}{c}\text { Matrix Effective } \\
\text { Young's } \\
\text { modulus } \\
\mathbf{( G P a )}\end{array}$ & $\begin{array}{c}\text { Matrix Effective } \\
\text { Shear modulus } \\
\mathbf{( G P a )}\end{array}$ & $\begin{array}{c}\text { Matrix Effective } \\
\text { Poisson's Ratio }\end{array}$ \\
\hline 0 & 1.63 & 0.51 & 0.42 \\
\hline 1.5 & 2.87 & 0.96 & 0.39 \\
\hline 5 & 5.88 & 2.08 & 0.37 \\
\hline 15 & 17.09 & 6.31 & 0.34 \\
\hline
\end{tabular}
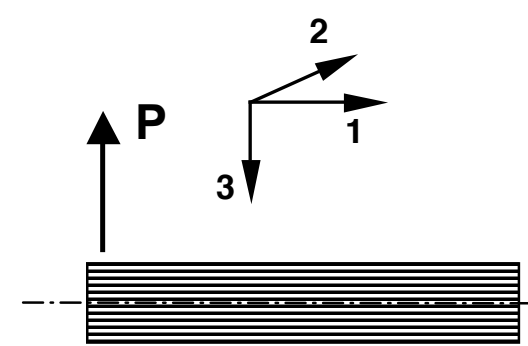

laminate midplane

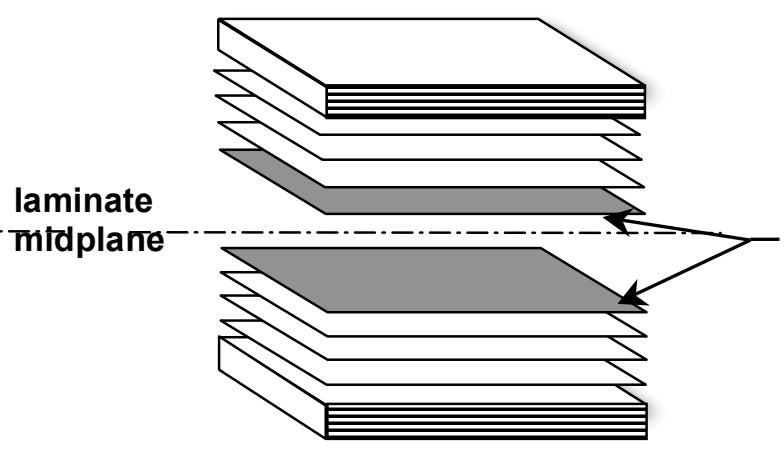

fiber-epoxy-CNT lamina

(a)

(b)

Figure 1. (a) Idealization of laminated carbon fiber-epoxy laminate and (b) exploded view depicting innermost lamina with carbon nanotubes.

(a)

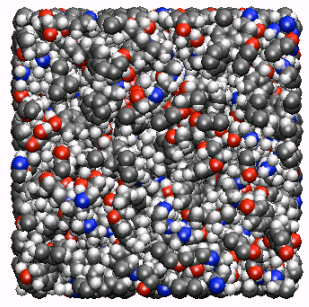

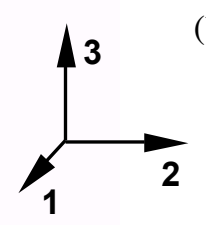

(b)

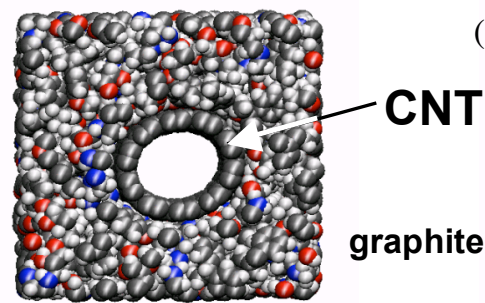

(c)

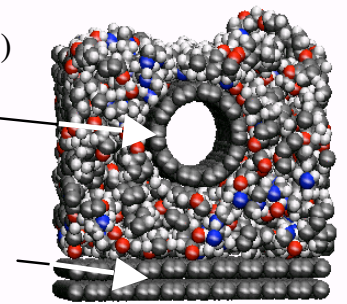

Figure 2. (a) Epoxy, (b) epoxy nanotube composite, and (c) epoxy nanotube and graphite. 


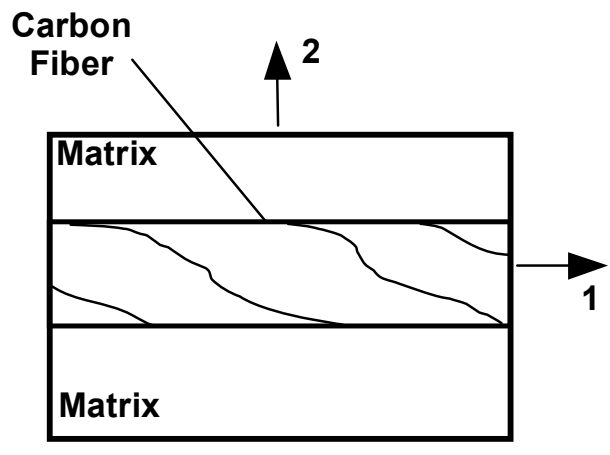

(a)

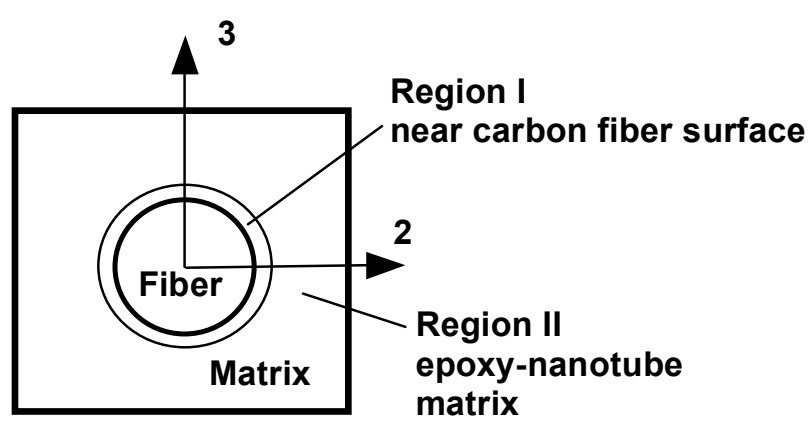

(b)

Figure 3. (a) Idealization of the carbon fiber/epoxy/CNT RVE and (b) cross-section view.

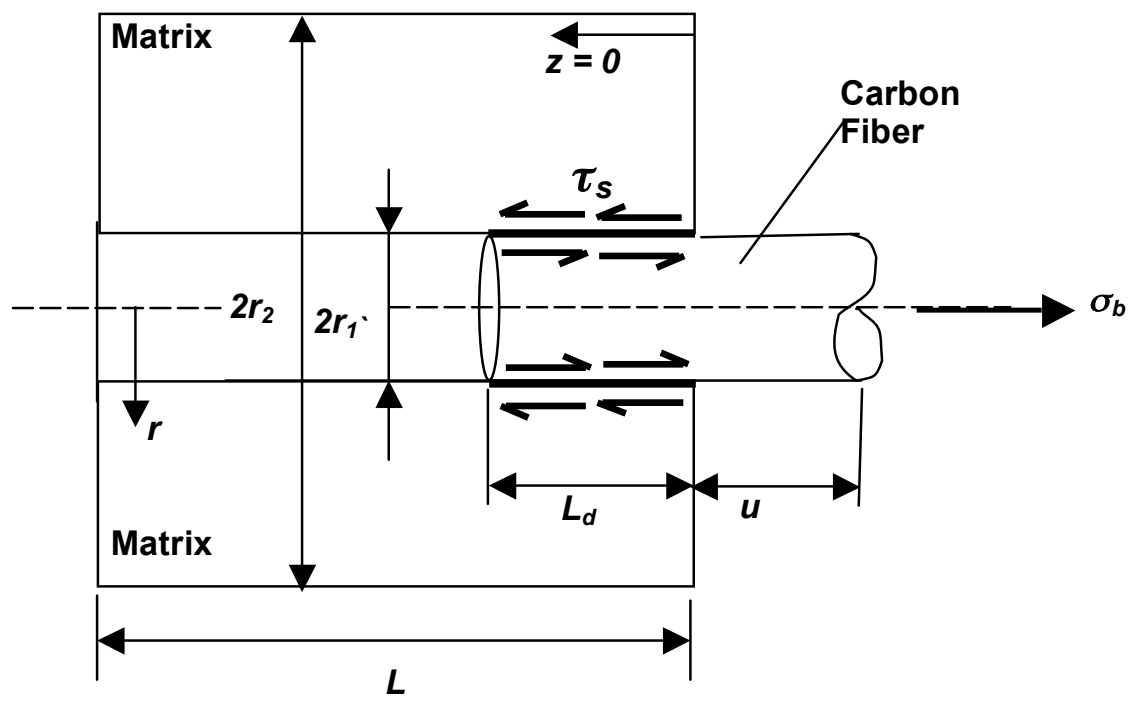

Figure 4. Geometry and nomeclature of the debonded interface under pull-out stress.
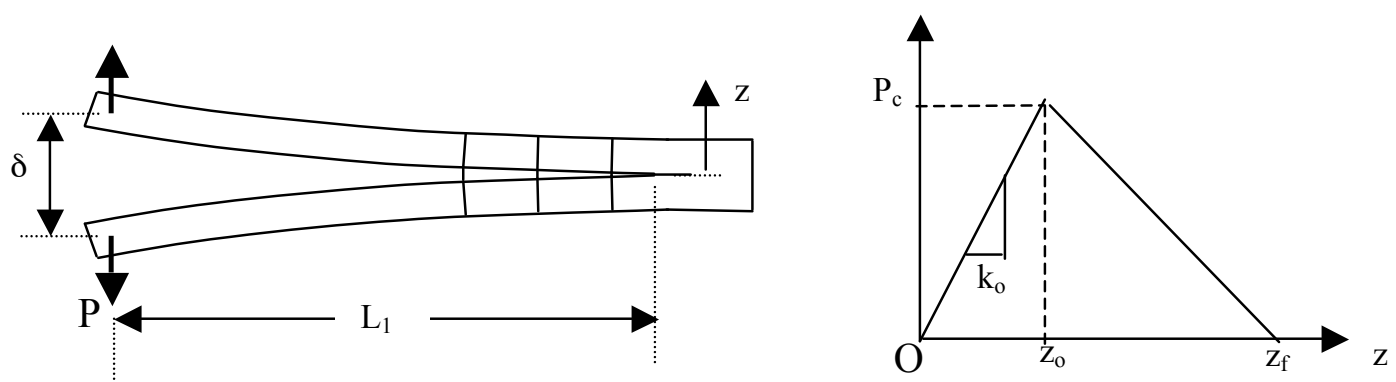

Figure 5. (a) Schematic of DCB specimen showing and (b) idealized damage for fiber pull-out. 


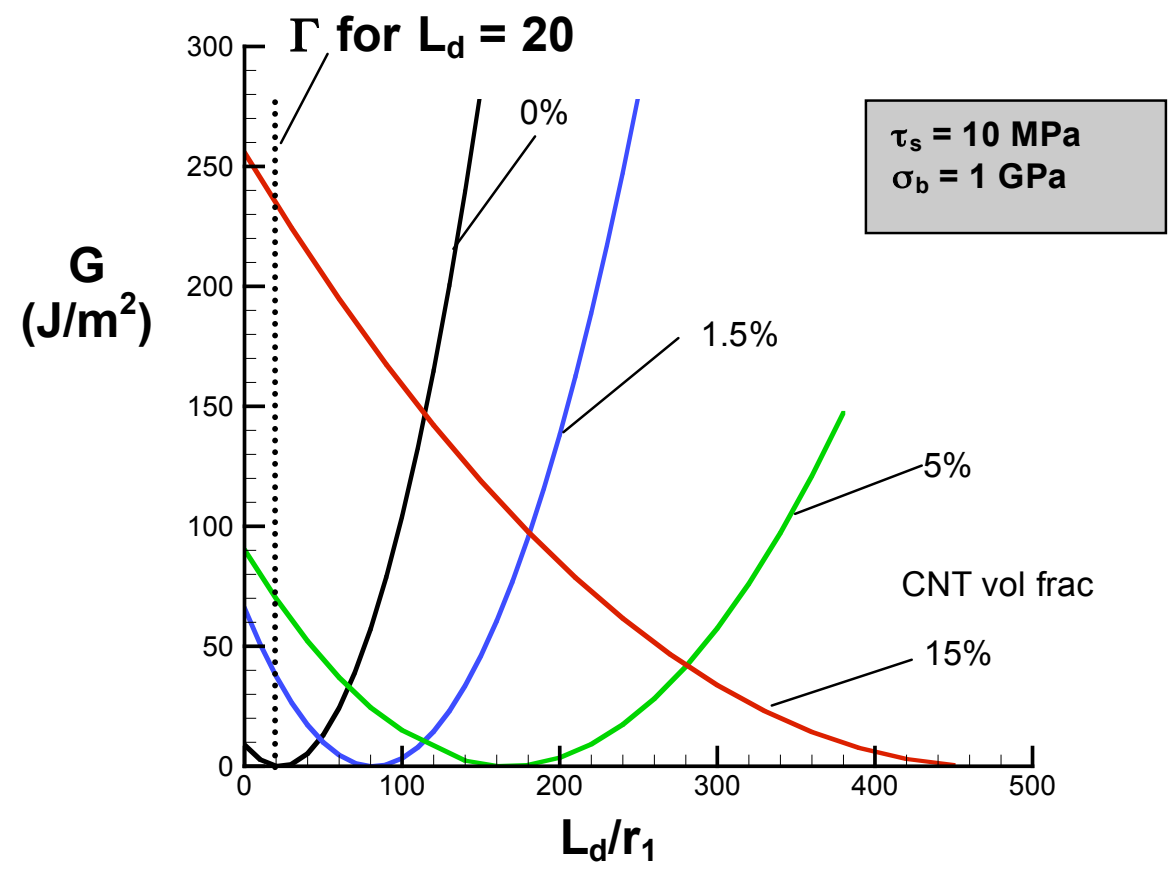

Figure 6. Distributions of strain energy release rate $G$ versus normalized debond length $L_{d} / r_{1}$ for various CNT volume concentrations.

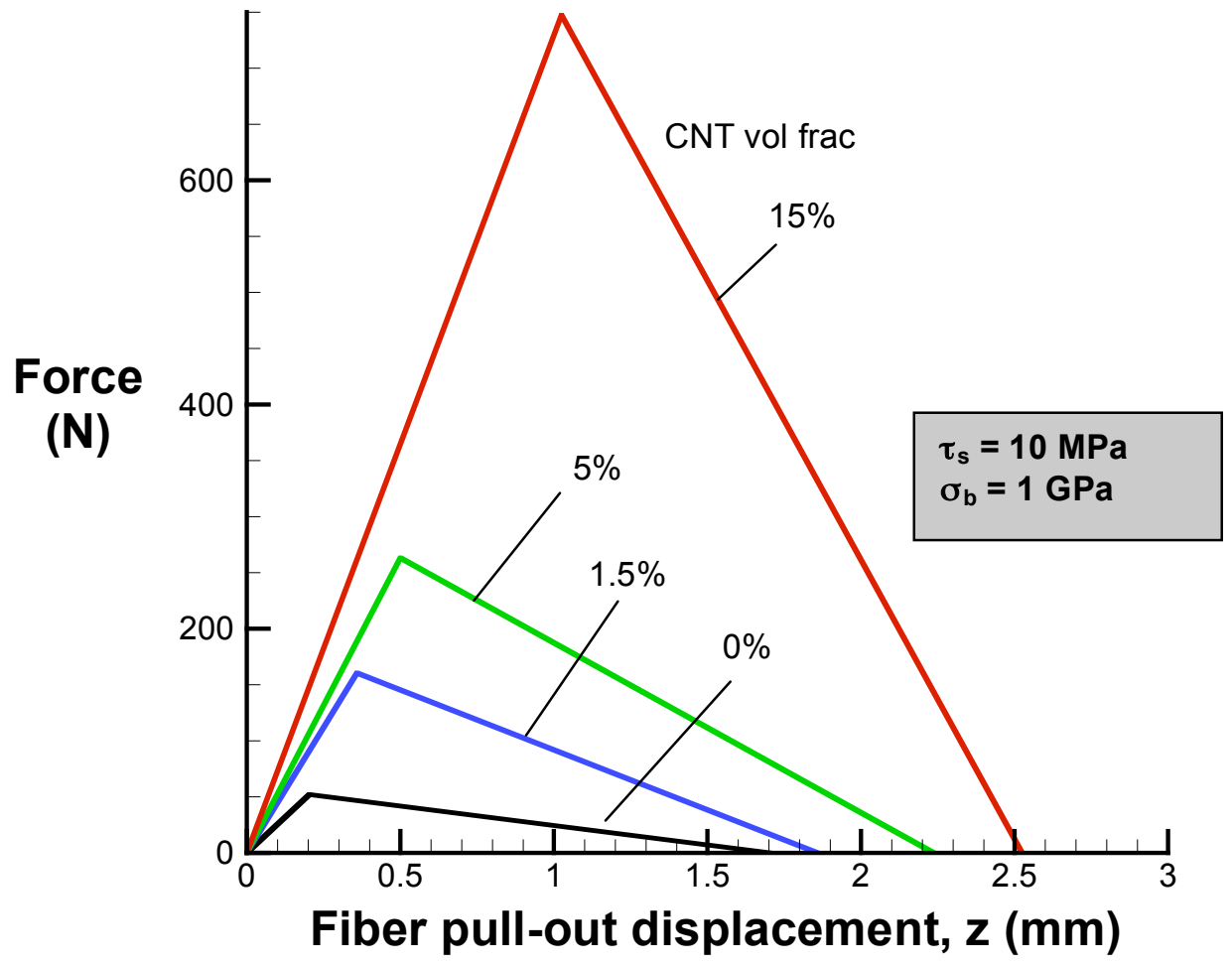

Figure 7. Traction laws governing fiber pull-out for various CNT volume concentrations. 


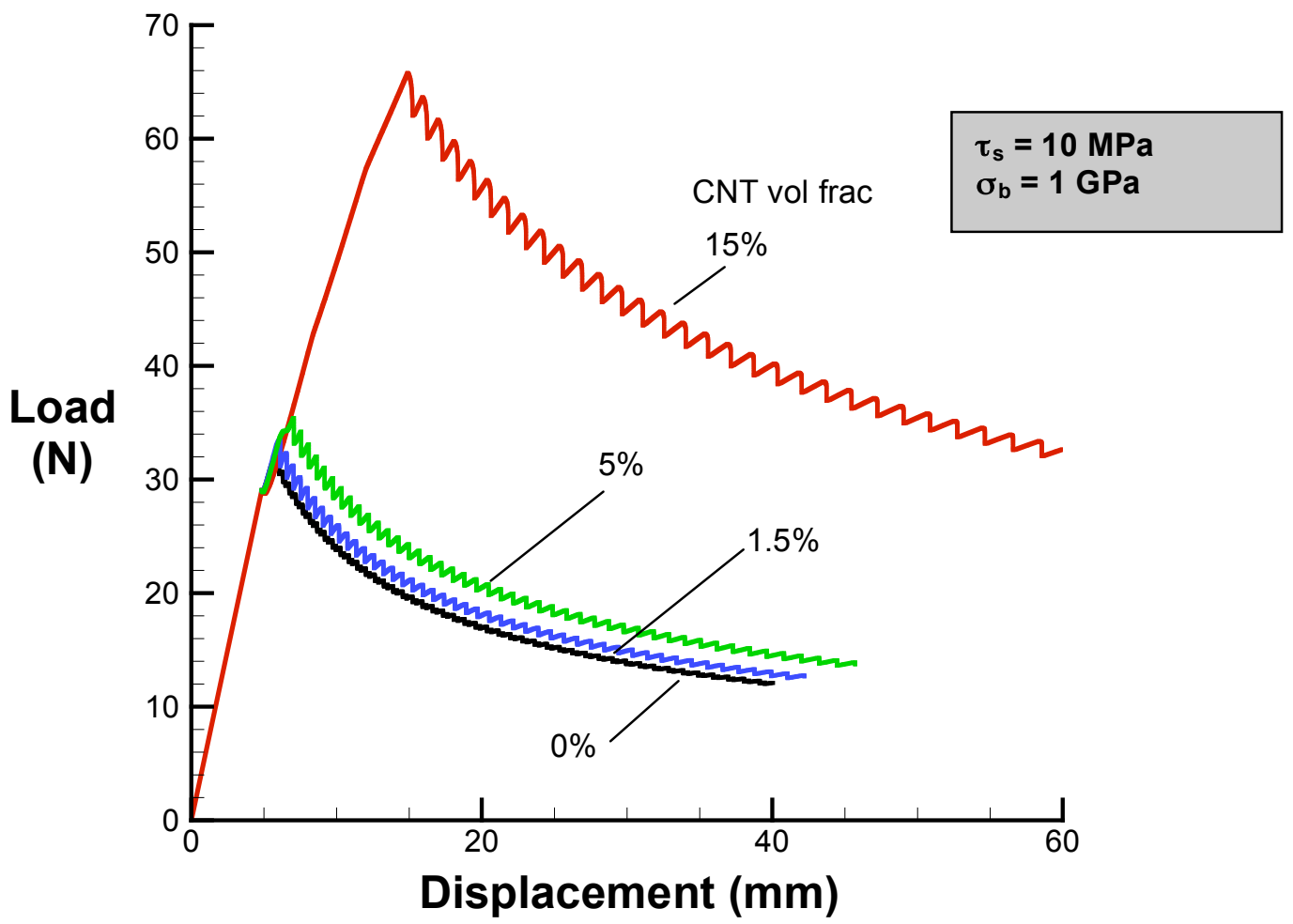

Figure 8. Estimated load-displacement response of idealized DCB specimens of various CNT volume concentrations. 